\title{
Diferenciación sexual y diferenciación social: su importancia en las variaciones morfológicas de los retratos de la Tumba Tebana 49, Egipto
}

\section{Judith E. Charlin", Liliana M. Manzi" y Marcelo Cardillo"}

Recibido:

1 de agosto de 2019

Aceptado:

8 de octubre de 2019

\section{Resumen}

En la decoración parietal de la Tumba Tebana 49, su propietario, Neferhotep, está representado junto con otros actores sociales desempeñando distintas actividades durante su vida terrena y en su proyección póstuma. Puede esperarse que algunas de estas expresiones visuales guarden altos grados de similitud con la realidad, mientras que otras se ajusten a una recreación de potenciales situaciones que ocurrirían a futuro, una vez que hubiese muerto. Al respecto, nos preguntamos si existe alguna clase de diferenciación en la forma de las representaciones que permita individualizar a las personas retratadas, o si sólo interesaba representar a aquellos grupos sociales que debían intervenir en los rituales de enterramiento. Persiguiendo este objetivo, nos proponemos evaluar la existencia de variaciones en la forma de los perfiles de las personas representadas, retomando algunos de los resultados alcanzados en trabajos precedentes. Dichos análisis, efectuados mediante las técnicas de morfometría geométrica, mostraron la existencia de diferencias dependientes del sexo como así también del estatus y/o rango social en los modos de representación de los perfiles de los personajes. Aquí nos interesa evaluar la interrelación de ambas variables y la importancia relativa del sexo (determinado mediante rasgos fisonómicos) en las variaciones morfológicas observadas en los retratos de hombres y mujeres.

\section{Sexual and social differentiation: their role in the morphological variations of portray scenes at Theban Tomb 49, Egypt}

\section{Palabras clave}

Tumba de Neferhotep Perfiles

Sexo

Estatus

Morfometría geométrica

\section{Keywords}

Neferhotep's Tomb Profiles Sex Status

Geometric morphometrics 
existence of shape variations in portrayed people profiles, according to previous geometric morphometric analyses that showed the existence of sex and rank and/or status shape differences. Here we are interested in assessing the interaction of both variables and the relative importance of sex (determined through of physiognomy) in morphological variations of male and female portrays.

\section{Introducción}

Neferhotep fue un miembro de la elite tebana de fines de la dinastía XVIII (15501307 a.C., Reino Nuevo) que se desempeñó como escriba en el templo de Amón en Karnak. En su carácter de propietario de la tumba tebana 49 (TT49) está representado desempeñando diferentes actividades como funcionario del estado y participando en distintas celebraciones, como así también se lo presenta en una proyección póstuma, durante sus propios ritos de enterramiento. En esas escenas se lo encuentra en relación con otros actores sociales, tales como sus parientes, representantes del poder político y miembros del cortejo fúnebre (Pereyra et al., 2006). Puede esperarse que algunas de estas expresiones visuales guarden alguna similitud con la realidad terrenal, mientras que otras sean una recreación de situaciones que ocurrirían a futuro, una vez que Neferhotep hubiera muerto. En ambos casos, nos preguntamos si existe alguna clase de diferenciación en las representaciones que permita individualizar a las personas retratadas, dando cuenta de individuos que formaron parte del universo social del propietario de la tumba, o si más bien lo que interesaba era representar a los grupos sociales (bajo cierto anonimato individual) que debían intervenir en los rituales de enterramiento en las distintas instancias de su desempeño de acuerdo con su estatus social. Para evaluar la existencia de diferencias morfológicas en los modos de representar a los individuos según el sexo y rango y/o estatus se estudió la forma de los perfiles de las personas retratadas en la TT49 a partir de métodos estadísticos multivariados (morfometría geométrica, ver infra), una práctica poco usual en la Egiptología y en los estudios iconográficos en general.

En el conjunto de personas representadas en la decoración parietal de la TT49 se observa al propietario de la tumba —en su carácter de figura central- desarrollando distintas acciones en diferentes momentos de su vida (Pereyra, 2012a). Tal es así que en algunas imágenes se lo observa como una persona adulta, mientras que en otras se lo presenta como un anciano (Figura 1).

Tanto en referencia a él como a sus parientes se cuenta con inscripciones jeroglíficas que permiten reconocer grados de parentesco, nombres y algunos títulos. De este modo se logra una identificación figurativa y textual de su esposa Merytra, su padre Neby, su madre Iwy, su abuelo Ptahemheb y su bisabuelo Nebbuneb. Los títulos de los ancestros masculinos muestran que la familia estaba vinculada a una tradición de escribas que servía en el templo de Amón en Karnak (Pereyra et al., 2006).

\section{Sectores, temáticas y actores sociales representados en la decoración funeraria}

En el antiguo Egipto la muerte fue concebida como una contra-imagen de la vida (Assmann, 2005), motivo por el cual se debía no sólo conservar el cuerpo sino también proporcionar referencias de posicionamientos sociales y desempeños, creando textual y visualmente fórmulas capaces de conjurar la aniquilación del individuo y dotarlo de recursos materiales e inmateriales que le permitieran alcanzar una dimensión eterna (Pereyra, 2011a; Yomaha, 2009). 

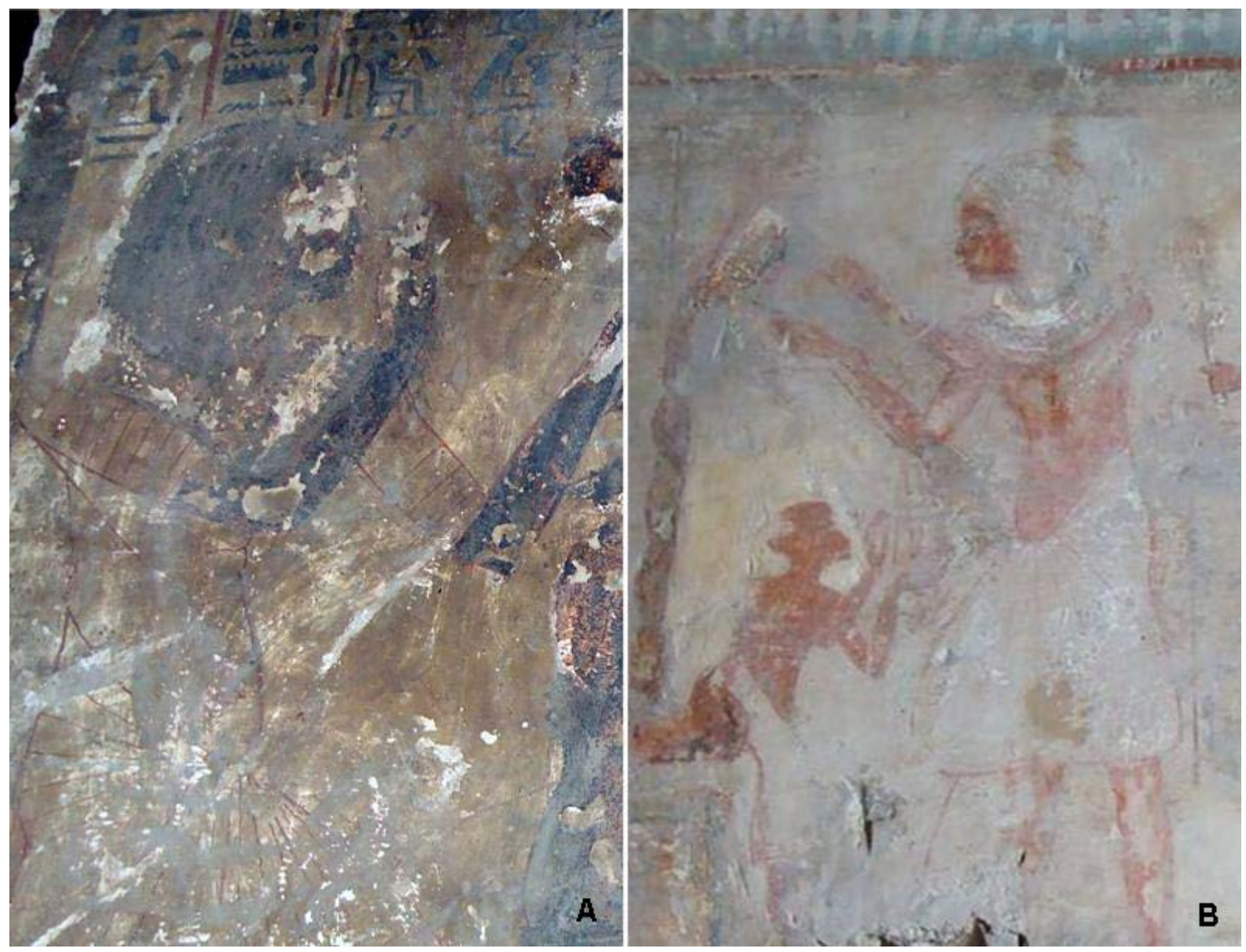

Figura 1. Retratos de Neferhotep: A) adulto y B) anciano.

La tumba es una ofrenda que el rey otorga (Faulkner, 1976, p. 179), donde las divinidades son invocadas en favor del difunto (Parra Ortiz, 2011, p. 137), a la vez que contiene las entregas materiales y otras clases de ofrendas representadas en las expresiones parietales (Assmann, 2004, 2005). La construcción y decoración del monumento fueron parte de estrategias creativas planificadas y ejecutadas por el estado (Hartwig, 2003; Manniche, [1987]1989; Staring, 2008). En consecuencia y ante esta supervisión, se espera que las expresiones iconográficas relacionadas con personas específicas y miembros de determinados grupos sociales evidencien escasas variaciones, más allá del hecho de que la creación presente una combinación de elementos reales con otros ideales (Laboury, 2009, p. 264, 2010, p. 2).

La tumba de Neferhotep corresponde cronológicamente al reinado de Ay (1327-1323 a.C.), de acuerdo con una cartela preservada en el vestíbulo. Las escenas parietales se distribuyen en relación a los distintos sectores que presenta el plano arquitectónico (Figura 2) con significación específica en la práctica ritual (Assmann, 2004). Cada uno de ellos se relaciona con temáticas particulares que se resumen a continuación a partir de los trabajos publicados al respecto (ver Fantechi, 2017; Fantechi y Zingarelli, 2003, 2006, 2009; Pereyra, 2011b, 2012b; Pereyra, Fantechi y Zingarelli, 2007; Pereyra et al., 2006; Pereyra y Zingarelli, 2004; Zingarelli, 2005).

En el patio (sector 1) se encuentra la primera mención a los títulos y nombre del propietario de la tumba. La fachada se compone de dos estelas verticales, una a cada lado de la puerta. En el campo superior de la ubicada en el lado sur se observa una 
escena doble de adoración, mientras que la estela del lado norte está sin terminar. En el pasaje exterior (sector 2) está representado Neferhotep ingresando y saliendo de la tumba junto con Merytra (paredes norte y sur, respectivamente).

En las paredes occidentales del vestíbulo (sector 3), lado sur, se encuentra la escena de la recompensa del propietario de la tumba y de su mujer de manos del faraón y su esposa. En las paredes norte y sur se observa la doble adoración a Osiris y Anubis, en la que los dioses reciben las ofrendas entregadas por Neferhotep. En los paneles laterales, el propietario de la tumba y su esposa son representados sedentes frente a mesas de ofrendas. En la pared este, se representa la preparación del ajuar y la procesión funeraria, junto a rituales de enterramiento y sobre el dintel se localizan dos siluetas de Anubis, mientras las paredes orientales exhiben escenas del paso de la vida terrenal al más allá.

Las escenas ubicadas en el segundo pasaje (sector 4) aluden al paso de la esfera terrenal al inframundo. En la pared sur los padres reciben a Neferhotep en el más allá. En la norte está representada la ofrenda de pan y agua que la diosa árbol brinda a la pareja, mientras que las aves y mariposas en vuelo, registradas en el cielorraso, aluden a la idea de renacimiento.

La capilla de culto, conformada por la sala de los pilares (sector 5), presenta en la pared norte las actividades oficiales de desempeño de Neferhotep en el ámbito del templo de Amón en Karnak. Las restantes paredes y los pilares registran expresiones de la devoción del propietario y su mujer a los dioses funerarios (Ra Harajty, Osiris, Anubis y Amenhotep I y su madre Ahmes Nefertary). Mientras que en la pared este se ofrenda al sol naciente, la pared oeste está dedicada a las divinidades funerarias y a la integración del difunto en el inframundo.

En la capilla de culto del $\mathrm{Ka}$ (sector 6) hay tres nichos; cada uno de ellos contiene parejas de esculturas sedentes. La central es la que corresponde a Neferhotep y Merytra. En torno a ellas se ubican escenas relacionadas con la celebración del culto funerario.

En el conjunto de representaciones pueden ser identificados distintos actores sociales (Figura 3). Algunos de ellos corresponden a su parentela, otros son representantes del poder político (el faraón y su esposa), mientras que algunos individuos se mantienen anónimos, pero tienen el potencial de ser identificados como grupos sociales con relevancia en los ritos funerarios (Pereyra et al., 2007; Zingarelli y Fantechi, 2014). Entre éstos se reconocen músicos, lloronas, portadores de ofrendas, etc. (Davies, 1933; Pereyra et al., 2006).

\section{Antecedentes}

En trabajos previos se exploró la aplicación de las técnicas de morfometría geométrica para el estudio de las variaciones morfológicas de las representaciones de la TT49, con diferentes objetivos (Charlin y Manzi, 2017, 2018; Pereyra, Manzi y Charlin, 2016). En primera instancia se analizaron los retratos de Neferhotep $(n=22$, tomados de Davies, 1973, vol. I; Pereyra et al., 2006) ubicados en las paredes norte y sur de la tumba, a partir de la idea de que podrían encontrarse variaciones a causa del desempeño de distintas cuadrillas de artesanos en la producción de las escenas que conforman la decoración de la tumba (Carpiceci, 2009; Choisy, 2007; Hermoso Cuesta, 2008).

Los resultados obtenidos no arrojaron diferencias estadísticas significativas entre los retratos de Neferhotep realizados en las distintas paredes, lo cual sugiere una alta uniformidad representativa que no estaría dando cuenta de la existencia de diferentes 


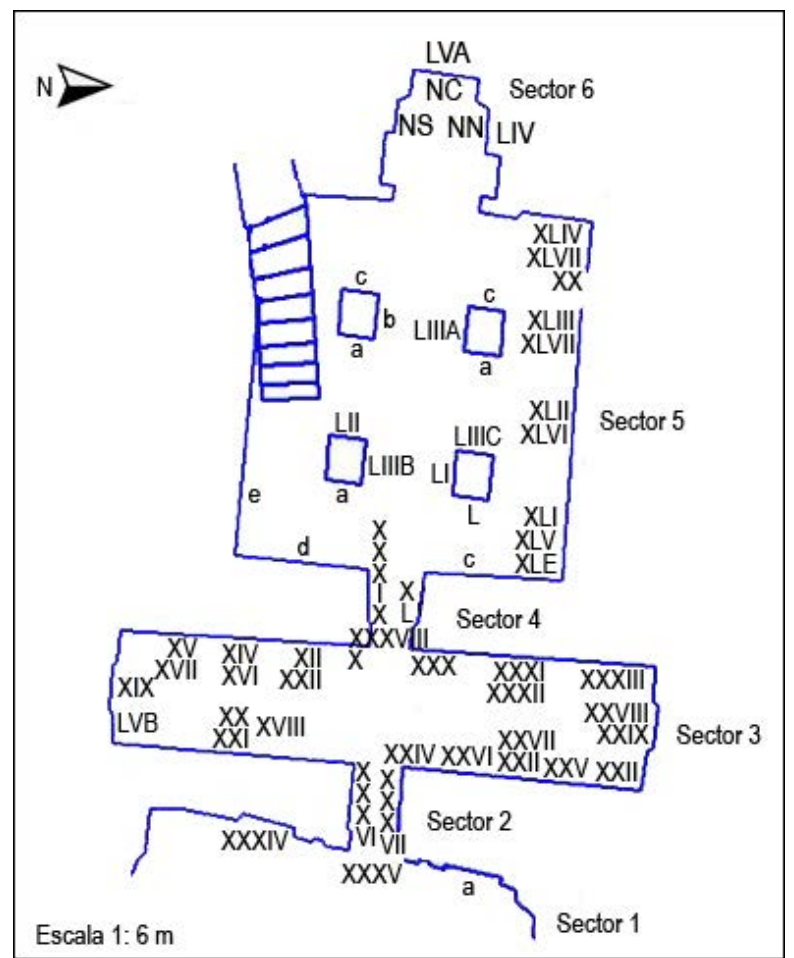

Figura 2. Sectores de la TT49 y ubicación de las escenas registradas: relevamiento de Davies (1933: I, pl. VI) en números romanos y de la Misión Argentina en Luxor (Pereyra et al., 2006) en alfabeto latino.
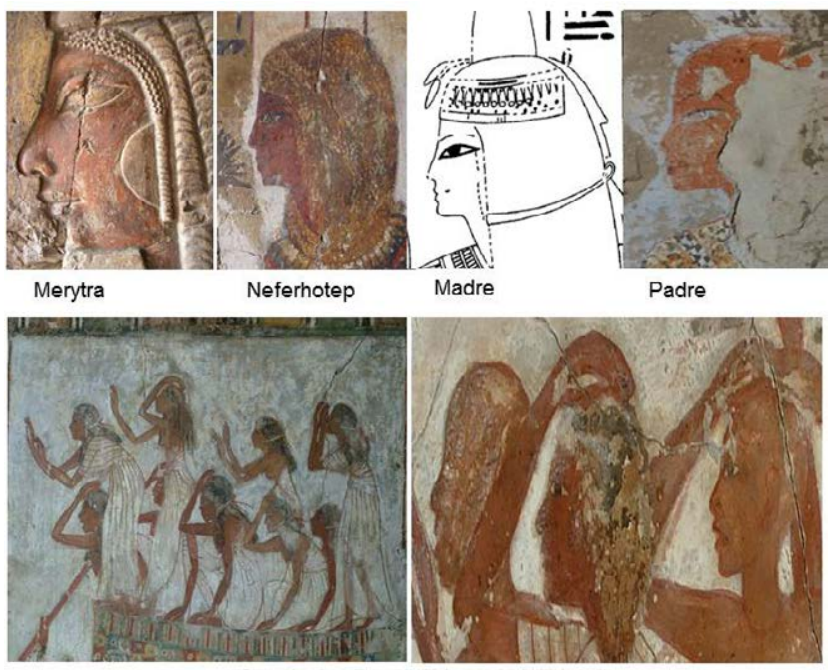

Cortejo de elite: plañideras / plañideros

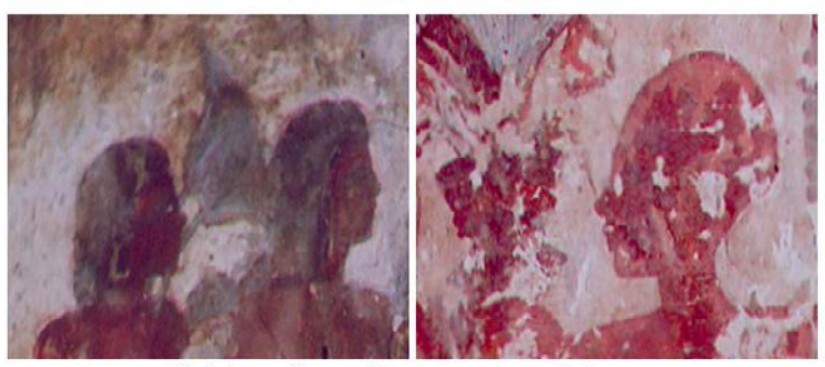

Cortejo no elite: servidores, remeros, portadores

Figura 3. Muestra de diferentes actores sociales presentes en las escenas de TT49 tomando a Neferhotep y su familia como grupo parental. 
cuadrillas de artesanos, al menos a partir del análisis de las variaciones morfológicas de los retratos del dueño de la tumba (Charlin y Manzi, 2017; Pereyra et al., 2016). Sin embargo, para descartar plenamente esta hipótesis es necesario poner en juego distintas líneas de evidencia. Al respecto, nos preguntamos si de existir cuadrillas de artesanos, hubo una especialización en lo que cada una de éstas realizaba, es decir, en los modos de representar, lo que dificultaría que se pudiera diferenciar realmente su accionar. En este sentido, no estamos exentos de situaciones de equifinalidad si consideramos que la ausencia de variaciones notorias en las representaciones del dueño de la tumba responde a un programa decorativo altamente pautado, que minimizaría los márgenes de variación a causa de una fuerte supervisión y una alta especialización, a pesar de la posible existencia de cuadrillas. Asimismo, por tratarse de retratos, sobre todo del dueño de la tumba, se espera que la variación tolerada fuese escasa, al estar dirigida al reconocimiento de rasgos fisonómicos identificatorios y su elaboración formalizada en un número variable pero limitado de alternativas representativas (Charlin y Manzi, 2017; Manzi, 2016).

En segunda instancia evaluamos la existencia de variaciones en la forma de los perfiles de los diferentes individuos presentes en la decoración mural $(n=88)$ buscando detectar semejanzas y diferencias morfológicas en dos casos en particular: 1) entre retratos femeninos y masculinos y 2) entre los retratos de los personajes agrupados por categoría social o rango.

En el primer caso se diferenciaron dos grupos conformados por 60 hombres y 28 mujeres con el fin de evaluar la existencia de cánones de representación dependientes del sexo, identificado a priori desde una perspectiva fisonómica. En el segundo caso, los actores sociales representados fueron clasificados en tres grandes grupos definidos de forma operativa según las tareas que los individuos se encuentran desempeñando en las escenas y que dan cuenta de su rango o estatus en una sociedad fuertemente jerarquizada (ver Katary, 2009 - para una división en subclases según ocupaciones en el papiro Wilbour-y Lemos, 2017 —para una diferenciación de perfiles sociológicos en elite, sub-elite y no elite según la cultura material de los entierros-). En dicha diferenciación se excluyó la figura de Amenhotep por tratarse de un personaje divinizado que no se corresponde con ninguno de los grupos diferenciados, siendo además un solo caso.

Tales grupos fueron $(\mathrm{n}=87$ perfiles):

1. Propietario de la tumba y familia

2. Integrantes del cortejo fúnebre (elite tebana)

\section{Servidores (no elite)}

El grupo 1 está compuesto por los retratos de Neferhotep, que son los predominantes, y de su familia, entre los que se encuentran su esposa Merytra, sus padres y su abuelo. Dicho grupo suma un total de 33 retratos, de los cuales 22 corresponden al dueño de la tumba. Por otro lado, el segundo grupo reúne a diferentes personajes de la elite tebana, como ritualistas y sacerdotes, plañidera/os, borrachas y músicas que forman parte del cortejo fúnebre. Este grupo reúne a 21 individuos pertenecientes a la elite. Finalmente, el grupo $3(n=33)$ se encuentra compuesto por los servidores y agrupa a figuras como los portadores de objetos y ofrendas, los remeros y las doncellas, que no forman parte de la elite tebana.

Los análisis discriminantes (para dos o más grupos) realizados sobre las variables de forma en los grupos diferenciados por sexo y por rango y/o estatus mostraron 
diferencias significativas en ambos casos (Charlin y Manzi, 2018). Sin embargo, nos interesa destacar aquí que si consideramos los casos de clasificación incorrecta de los individuos (si se los toma como "desconocidos" o "indiferenciados" en la variable de grupo definida a priori), los porcentajes de error son mayores para los casos de los grupos diferenciados por rango y/o estatus (entre un 40 y 56\% aproximadamente según función discriminante y validación cruzada respectivamente), lo cual parece sugerir que el sexo es un marcador más claro en la diferenciación de los individuos representados en la decoración funeraria (con sólo un 14 y 34\% de error, ver detalles en Charlin y Manzi, 2018).

\section{Objetivos}

En los análisis realizados en estudios previos las variables sexo y rango o estatus utilizadas para definir los grupos fueron tratadas de forma independiente, conformándose grupos diferenciados por sexo o por estatus de forma separada, interviniendo en diferentes análisis. A continuación nos proponemos evaluar la interrelación de ambas variables en las estrategias de representación y la importancia relativa de las variaciones dependientes del sexo, en tanto parece ser el marcador más distintivo en la diferenciación de grupos en comparación con la adscripción social de los individuos representados.

\section{Materiales y métodos}

En el presente trabajo se utilizan las variables de forma (agrupadas en componentes principales) obtenidas del análisis morfogeométrico de 87 imágenes de diferentes individuos representados en la decoración mural de la TT49 presentado en Charlin y Manzi (2018). Se emplean los tres primeros componentes principales de forma porque son los que explican las mayores variaciones morfológicas (medidas mediante coordenadas de Procrustes), dando cuenta de un $76,5 \%$ de la variación total de la muestra. Aquí retomamos las diferencias obtenidas previamente para evaluar la relación existente entre sexo y rango o estatus social en las variaciones morfológicas observadas en los retratos. Los mismos fueron relevados mediante fotografías digitales tomadas in situ (Dra. M. V. Pereyra) y láminas publicadas (Davies, 1933, vol. I; Pereyra et al., 2006) que fueron escaneadas para su inclusión en el análisis. Las figuras utilizadas se encuentran de perfil, dados los modos típicos de representación en el Egipto antiguo; todas han sido orientadas o reorientadas en el mismo sentido - hacia la izquierda - y haciendo coincidir el mentón con una línea horizontal ubicada a modo de base. Estos procedimientos fueron implementados para estandarizar la posición y traslación de las figuras, lo cual constituye un requisito del método.

En el presente trabajo no pretendemos hacer una síntesis de los principios fundamentales de la morfometría geométrica, ya que esto ha sido efectuado en numerosos trabajos previos, sólo destacamos que dicha metodología reúne un conjunto de métodos estadísticos para la descripción cuantitativa de la forma mediante el estudio de las relaciones espaciales entre puntos discretos representados por coordenadas cartesianas (Bookstein, 1982, 1990, 1991). Estos puntos son denominados landmarks y semilandmarks según sus propiedades (Bookstein, 1996-1997; Gunz y Mitteroecker, 2013). De esta manera, la forma se convierte en una variable numérica compuesta por coordenadas x,y para cada uno de los puntos considerados (también llamadas coordenadas de Procrustes), los cuales varían en número de acuerdo con el objeto de estudio y la problemática de interés. Un desarrollo detallado de las bases del método pueden consultarse en Bookstein (1991), Marcus, Bello y Garcia-Valdecasas (1993), Rohlf (1999), Rohlf y Bookstein (1990), Slice (2005), Zelditch, Swiderski, Sheets y David (2014), Zelditch, Swiderski, Sheets y Fink (2004), entre otros. Asimismo existen varios 
trabajos de revisión sobre el desarrollo de esta metodología relativamente recientes (Adams, Rohlf y Slice, 2013; Lawing y Polly, 2009; Mitteroecker y Gunz, 2009; Slice, 2007; Slice, Bookstein, Marcus y Rolhf, 2009; Viscosi y Cardini, 2011; Webstern y Sheets, 2010, entre otros). Un glosario de términos, conceptos y tipos de análisis más frecuentes en morfometría geométrica puede consultarse online en http://life. bio.sunysb.edu/morph/glossary/gloss1.htm y gloss2.htm (Slice et al., 2009). Para una introducción teórico-metodológica al método y su aplicación en arqueología consultar Cardillo (2010), Charlin y Hernández Llosas (2016), Okumura y Araujo (2019), Pérez (2003), Shott y Trail (2010, 2012), entre otros. La aplicación de la morfometría geométrica al estudio de las representaciones visuales en particular puede consultarse en Charlin y Hernández Llosas (2016), Cobden et al. (2017) y en los antecedentes de este estudio anteriormente citados. Numerosas aplicaciones de los métodos basados en landmarks (en 2D) sobre artefactos líticos se encuentran en Buchanan y Collard (2010), Buchanan, Collard, Hamilton y O'Brien (2011), Buchanan, Eren, Boulanger y O'Brien (2015), Cardillo (2009), Cardillo, Borrazzo y Charlin (2016), Cardillo y Charlin (2016), Castiñeira, Cardillo, Charlin y Baeza (2011), Castiñeira, Charlin, Cardillo y Baeza (2012), Charlin y Cardillo (2018), Charlin, Cardillo y Borrazzo (2014), Charlin y González-José (2012, 2018), Morales, Soto, Lorenzo y Vergés (2015), Okumura y Araujo (2014), Thulman (2012), Shott y Trail (2010, 2012), entre otros.

Como mencionamos anteriormente, en este trabajo utilizamos como variables de forma los tres primeros componentes principales obtenidos de los análisis efectuados en Charlin y Manzi (2018). En dicho trabajo, para describir y cuantificar la forma de los perfiles se usaron los métodos basados en landmarks, disponiendo un total de 18 puntos morfométricos (cinco landmarks y 13 semilandmarks) sobre los perfiles de los individuos retratados (Figura 4, ver detalles en Charlin y Manzi, 2017, 2018). Aquí, con el fin de explorar la relación entre las tendencias generales de forma para cada sexo y los grupos sociales diferenciados por rango y/o estatus, se realizó una regresión multivariada mediante un análisis de redundancia. El análisis de redundancia (Legendre y Legendre, 1998) es una extensión de la regresión lineal múltiple que genera un número reducido de nuevas variables que maximizan la correlación entre las variables dependientes (componentes de forma) y las variables explicativas (grupo social o rango). Estas nuevas variables se representan en un espacio de coordenadas en donde podemos visualizar la relación entre las variables y los casos de estudio (los retratos) y el porcentaje de la variación explicado por esta relación. De esta forma, se realizaron dos análisis de redundancia empleando los tres primeros componentes principales de forma, uno para el sexo masculino y otro para el femenino.

\section{Resultados}

El análisis de redundancia (RDA) realizado sobre los componentes de forma en retratos femeninos y masculinos es significativo para ambos sexos (ANOVA para retratos femeninos: $\mathrm{F}=2.5781, \mathrm{p}=0,028$; ANOVA para retratos masculinos, $\mathrm{F}=$ $4.0252, \mathrm{p}=0,008$ ). Entre los retratos femeninos, el sexo explica un $17 \%$ de la variación morfológica $\left(\mathrm{R}^{2}=0,17\right)$ observada en el grupo de las mujeres. De los dos primeros ejes de redundancia, el primero (RDA1) contiene el $94 \%$ de esta variación, tal como se observa en la Figura 5.

La Figura 5 señala que los retratos femeninos que fueron agrupados en la categoría "Familia" (compuestos por los retratos de Merytra, esposa de Neferhotep y de Iwy, su madre) están relacionados positivamente con el primer componente principal de forma y comprenden los casos con puntajes negativos del primer RDA. Los retratos de plañideras, músicas y borrachas que forman parte del "Cortejo," por otro lado, se correlacionan positivamente con el primer RDA y con el tercer componente principal de 


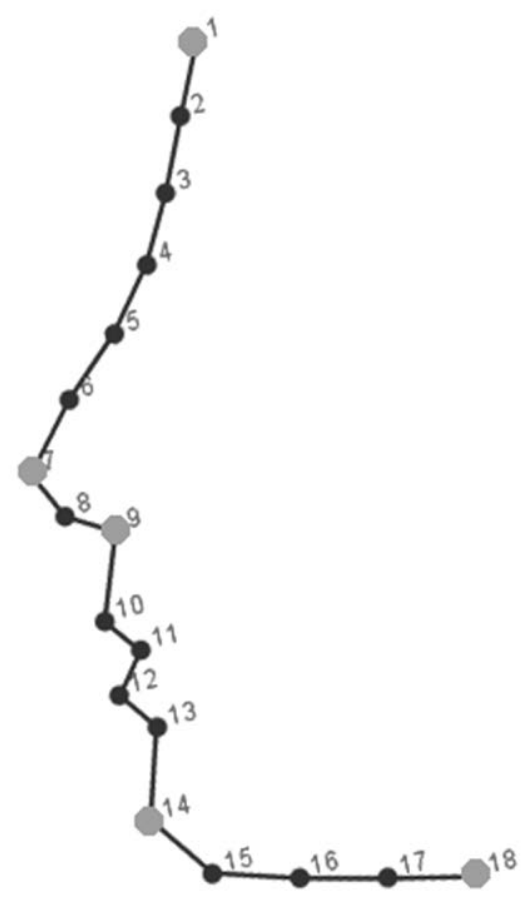

Figura 4. Localización de landmarks (círculos grises) y semilandmarks (círculos negros) en los perfiles retratados. Se muestra la forma media.

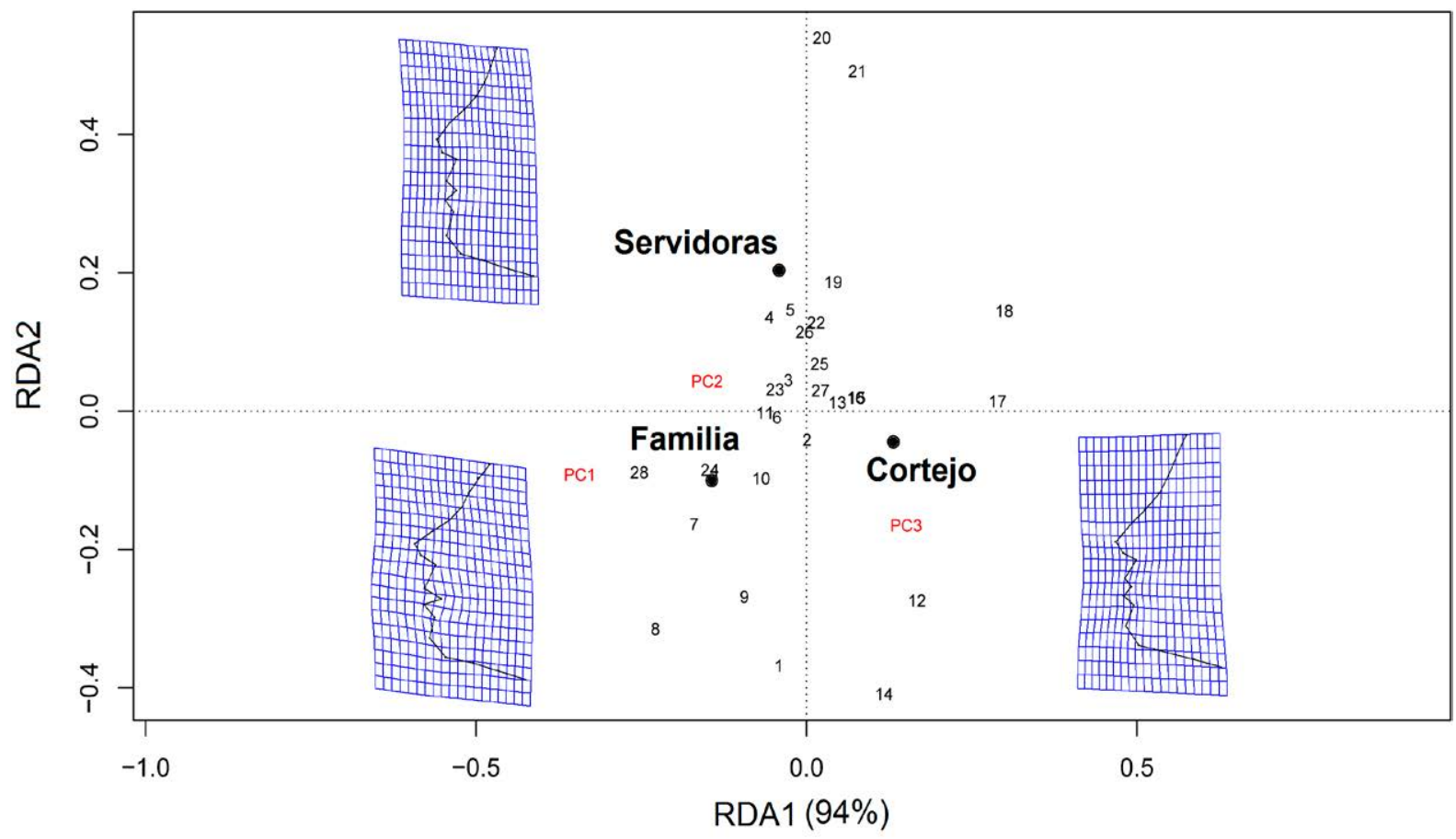

Figura 5. Análisis de redundancia sobre los componentes de forma en los retratos femeninos.

forma, abarcando los casos con puntajes positivos en el primer RDA. Las "Servidoras", por otro lado, se vinculan principalmente con los puntajes positivos del segundo RDA, el cual explica pocas variaciones $(5,03 \%)$ y con el segundo componente principal de forma. Por su distribución, los resultados sugieren que los retratos agrupados en "Familia" y "Cortejo" son los dos grupos morfológicos que más se diferencian entre 


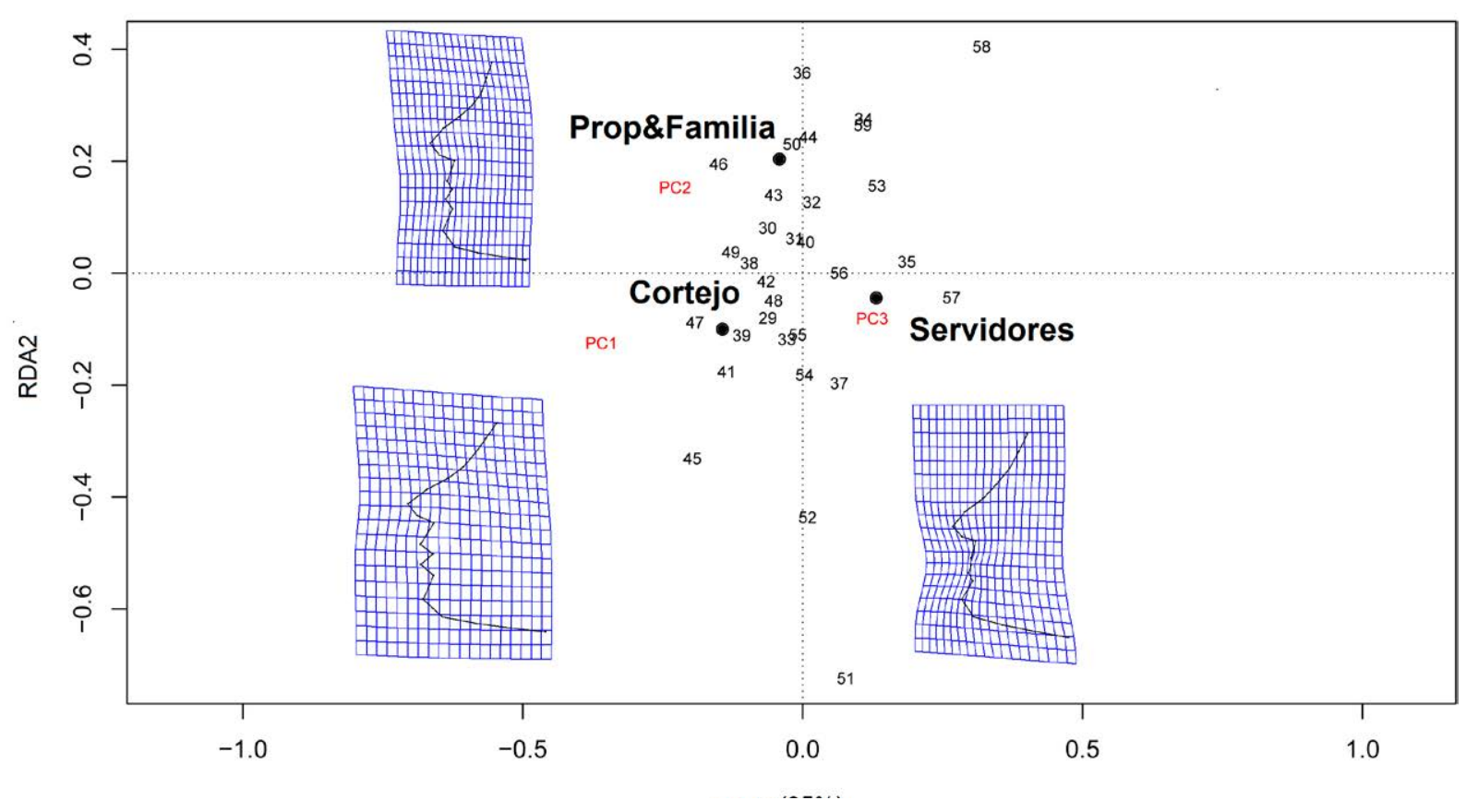

Figura 6. Análisis de redundancia sobre los componentes de forma en los retratos masculinos.

sí, lo que explica las mayores variaciones entre los retratos femeninos (94,97\%). Una proporción mucho menor de la variación se encuentra en el grupo de las "Servidoras".

En cuanto a los retratos masculinos, el sexo explica el $22 \%$ de la variación morfológica $\left(\mathrm{R}^{2}=0,22\right)$ dentro del grupo de los hombres. Como en el caso anterior, el primer eje (RDA1) da cuenta de las mayores variaciones (94,89\% del total de la variación), pero a diferencia de lo que sucede entre los retratos femeninos, aquí el "Cortejo" y los "Servidores" son los grupos más explicativos (Figura 6). Su distribución opuesta en el gráfico muestra que son los grupos con las mayores diferencias morfológicas. Los casos con puntajes negativos se vinculan a individuos del "Cortejo", en tanto que los positivos a los "Servidores". En cuanto a los componentes de forma, los "Servidores" están vinculados al tercer componente principal y el "Cortejo" al primero.

Con respecto a los retratos de Neferhotep, su padre y su abuelo agrupados en la categoría "Propietario de la tumba y familia" (Prop\&Familia en Figura 6), éstos se vinculan con el segundo RDA y con el segundo componente principal de forma (PC2) y dan cuenta de pocas variaciones (solamente un 5\%).

En síntesis, los resultados del RDA señalan cuatro tendencias principales en las variaciones morfológicas de los retratos. En primer lugar, los retratos muestran diferencias en la forma de los perfiles, esto es, en el modo de representación según el sexo. En segundo lugar, los retratos que conforman cada uno de los grupos que fueron diferenciados al interior de cada sexo según rango o estatus se correlacionan con distintos componentes de forma (tanto en los retratos femeninos como masculinos), lo cual sugiere la existencia de diferencias morfológicas entre los grupos de acuerdo con la jerarquía social (además del sexo). En tercer lugar, los retratos muestran distintos grados de variación al interior del grupo social diferenciado, es decir, algunos grupos son más homogéneos que otros (los retratos muestran mayores semejanzas entre sí), lo que explica diferentes porcentajes de la variación morfológica total para cada uno de los sexos. Así, en el caso de las mujeres "Cortejo" y "Familia" son los grupos que presentan la mayor variación morfológica, mientras que en el caso de los hombres la 
mayor diferencia está dada entre los casos asignados al "Cortejo" y a los "Servidores". Finalmente, el porcentaje de variación explicado en cada caso, sugiere que dentro de los grupos diferenciados por sexo hay un remanente de varianza de $83 \%$ para las mujeres y $78 \%$ para los hombres que no puede ser explicado por las diferencias sexuales.

\section{Discusión}

Entre los individuos representados en los murales de TT49 se reconocen hombres y mujeres según sus rasgos fisonómicos, a quienes también se los puede distribuir en relación a sus desempeños en cargos (funciones) y/o en rangos (estatus) en la sociedad tebana. Algunos personajes representados son miembros de la elite, con jerarquía acorde al posicionamiento social de Neferhotep, quien habría ejercido un cargo alto dentro de los escribas tebanos. Por este motivo se espera que las escenas que muestran su desempeño en vida y en las cuales se lo vincula con otros actores, estén relacionadas con el ejercicio de tareas y roles de acuerdo con su posicionamiento social y con el cumplimiento de acciones específicas dentro de la práctica ritual. En este sentido, habría una serie de restricciones creativas en cuanto a la composición de las escenas murales de la TT49 en referencia a la jerarquía y cargos del propietario, la selección de las actividades y acciones desarrolladas a lo largo de su vida, como así también en las formas en que se debieron representar a las personas en vida y después de muertas.

El culto a la memoria de los ancestros debió ser una parte importante de las celebraciones, realizadas tanto antes como después de depositada la momia del propietario. En este sentido, se explica la representación de su padre, madre y abuelo, quienes se supone que ya estaban fallecidos al momento de la construcción del monumento, dado que Neferhotep es representado como una persona mayor e incluso como anciano. Además son sus padres quienes lo reciben en el más allá (Pereyra et al., 2006; Zingarelli y Fantechi, 2014).

Las referencias a su familia directa son explícitas y permiten su individualización tanto iconográfica como textual, excepto su bisabuelo que no está representado pero si mencionado en una inscripción. En función de esta especificidad es esperable que hubiese artesanos especializados en esta clase de representaciones, como por ejemplo retratistas encargados de representar al propietario de la tumba y a su familia. Quizás los ya fallecidos (padre, madre, abuelos) fueran representados a partir de rasgos de semejanza con el pariente vivo, en tanto otros artesanos serían los encargados de retratar al resto de los personajes, fuera de la esfera parental pero formando parte necesaria de las escenas. Esto podría explicar las pocas variaciones observadas en los retratos de Neferhotep y sus parientes varones, a diferencia de los retratos masculinos de servidores y miembros del cortejo que muestran las mayores variaciones morfológicas. En el caso de los servidores se observan diferencias representativas que podrían responder a una convención para hacer alusión a su no pertenencia a la elite. En la Figura 7 puede observarse que entre los remeros, uno de ellos muestra rasgos negroides que bien podrían remitir a una posible procedencia desde Sudán, aunque en ausencia de convenciones representativas que aludan a la presencia de extranjeros - como bien podría ser el arreglo del cabello- este rasgo podría estar dando cuenta de la composición poblacional del Egipto antiguo ${ }^{1}$. Una situación diferente se observa entre los retratos femeninos de las servidoras, grupo conformado principalmente por doncellas que parecen reproducir la "apariencia" de Merytra, con pocas variaciones, aunque conformando un grupo independiente (Figura 7).

A diferencia de los parientes cercanos que es posible identificar, se observan actores sociales que, si bien son indispensables para la representación del ritual de enterramiento, permanecen anónimos, aunque su número y composición en grupos
1. La actual población de Egipto responde a la radiación del Islam en momentos más recientes. 


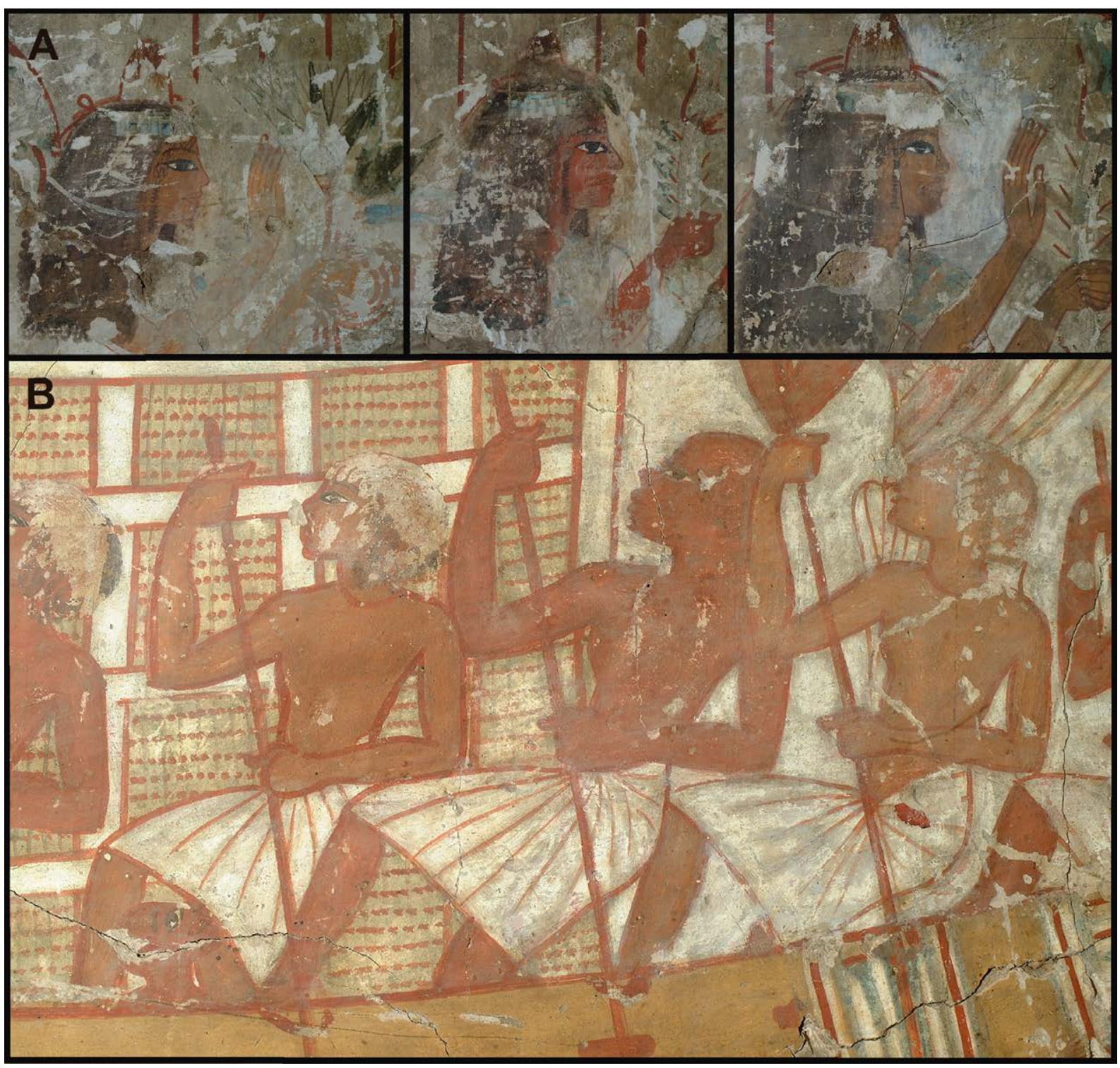

Figura 7. Variaciones morfológicas por rango en los retratos femeninos y masculinos. El caso de las doncellas $(A)$ y los remeros $(B)$ que conforman el grupo de los servidores. Referencias de las fotografías con respecto a dibujos publicados: $A$ ) se corresponden con la figura 24 de Pereyra y otros (2006); B) se corresponde con la lámina XXIII de Davies (1933).

debieron ser acordes con los honores rendidos con respecto al rango del difunto. Estos individuos "anónimos" en general han sido identificados como servidores y miembros del cortejo fúnebre según los roles desarrollados en las escenas. Algunos de ellos son miembros de la elite, mientras que otros son parte de la población egipcia que prestaba diversas clases de servicios, evidencia de la usual interacción entre miembros de la elite y no elite, como los servidores (Kemp, 1977; Spence, 2010). Comparaciones de tipos de tumbas según categorías sociales o perfiles sociológicos muestran grandes diferencias en términos de recursos y tiempo invertido entre miembros de la elite y sub-elite en relación a la no-elite (sensu Lemos, 2017), quienes serían parte del grupo de los servidores en nuestra diferenciación.

Los análisis aquí desarrollados señalan que los grupos de retratos conformados según el rango o estatus de los personajes representados se correlacionan con diferentes componentes de forma (los cuales son ortogonales, es decir, independientes). Este hecho 
sugiere que la forma de los perfiles muestra patrones diferenciales pero recurrentes que permiten distinguir actores sociales al interior de los grupos segmentados por sexo (ver también Charlin y Manzi, 2018), ofreciendo evidencia adicional a la discusión sobre cómo identificar jerarquías sociales en el antiguo Egipto (ver Katary, 2009; Lemos, 2017).

Si bien las variables consideradas para la segmentación en grupos (el sexo y el estatus y/o rango) se encuentran correlacionadas, las distinciones sociales explican diferentes porcentajes de la variación morfológica en los retratos femeninos y masculinos. Entre los primeros, son los retratos de la familia (Merytra e Iwy) y del cortejo los que explican las mayores variaciones, en tanto en los retratos masculinos éstas son explicadas por el cortejo y los servidores, mostrando los retratos de Neferhotep, su padre y su abuelo una mayor uniformidad. Sin embargo, si bien el sexo explica un $17 \%$ y $22 \%$ de las variaciones morfológicas en los retratos femeninos y masculinos respectivamente, un alto porcentaje de la variabilidad queda sin explicar. Al respecto consideramos que algunas de las expectativas comúnmente asumidas cuando se postula la existencia de especialización artesanal como prerrequisito para alcanzar una estandarización representativa deben ser repensadas en referencia a situaciones específicas. En general, cuando se espera la adhesión a convenciones que den como resultado una alta uniformidad morfológica o estilística (que puede ser entendida como una "escuela" o "taller" de artesanos) debe también esperarse que exista un margen que permita cierta individualización, circunstancia que origina variabilidad en formas estandarizadas. Es decir, existe suficiente estandarización en la representación de las formas que permite diferenciar a las personas retratadas por funciones y estatus, pero al mismo tiempo se observa cierto grado de laxitud en la representación de determinados personajes, de modo que puedan ser reconocidos e individualizados, aunque formen parte de un mismo colectivo.

Si bien los análisis aquí presentados muestran la existencia de estandarización de formas que puede atribuirse a la especialización artesanal, ya que es posible la conformación de grupos con cierta homogeneidad interna que permite diferenciarlos de otros, es necesario replantear la escala de estas variaciones. En el caso aquí presentado, la especialización artesanal parece expresarse en el alto grado de detalle, lo cual a su vez se manifiesta en "rasgos estilísticos únicos" que incrementan la variabilidad y favorecen la individualización (ver Sapirstein, 2013 para estos casos en la decoración de la cerámica griega). Estas distinciones aumentan si tomamos en cuenta los gestos, acciones, vestimenta, adornos, equipamiento y tamaño relativo de las figuras, entre otros elementos que no fueron incluidos en este análisis, debido al requisito de homología entre los rasgos comparados, motivo por el cual solamente fueron estudiados los perfiles.

\section{Conclusiones}

La forma de los perfiles de los distintos personajes representados en las diferentes escenas de la TT49 muestra variaciones que permiten diferenciar los retratos tanto por sexo como por rango y/o estatus social según su morfología, explicando distintos porcentajes de la variación según se trate de retratos femeninos o masculinos. Así, es posible sostener que la representación de la mayoría de los grupos está altamente pautada a través de un diseño recurrente tanto de los perfiles como de otros atributos como gestos, acciones, vestimenta, adornos, objetos portados, entre otros. Sin embargo, si bien en estudios previos el sexo mostró ser la variable con menor error en la clasificación de los individuos, los análisis aquí presentados señalan que gran parte de las variaciones morfológicas observadas no son explicadas por estas diferencias. $\mathrm{Al}$ respecto, otros factores deberán ser considerados en futuros trabajos para lograr 
comprender de forma integrada la variabilidad morfológica expresada en los retratos, tales como las diferencias etarias (Pereyra, 2017), entre otros. Finalmente, cabe señalar que los resultados estadísticos aquí presentados aplicando las técnicas de morfometría geométrica a las representaciones parietales (pinturas y bajo relieves) han mostrado su potencial para contribuir a los debates sobre la diferenciación de jerarquías sociales en el antiguo Egipto.

\section{Agradecimientos}

Estas investigaciones se enmarcan en el proyecto Programación Científica UBACyT 20020170100688BA (2018-2020) "Registros funerarios en el Cercano Oriente antiguo: análisis de estructuras, representaciones e inscripciones", dirigido por la Dra. L. Manzi. Nuestros agradecimientos están dirigidos a la Dra. V. Pereyra quien revisó una versión previa de este trabajo y a los evaluadores anónimos de este manuscrito que mejoraron notablemente su calidad gracias a sus comentarios y sugerencias bibliográficas. 


\section{Referencias citadas}

"Adams, D., Rohlf, F. y Slice, D. (2013). A field comes of age: Geometric morphometrics in the 21th century. Hytrix, the Italian Journal of Mammalogy, 24(1), 7-14.

" Assmann, J. (2004). The Ramesside Tomb and the Construction of Sacred Space. En N. Strudwick y J. Taylor (Eds.), The Theban Necropolis. Past, Present and Future (pp. 46-52). London: British Museum.

》 Assmann, J. (2005). Death and Salvation. Ithaca: Cornell University Press.

" Bookstein, F. (1982). Foundation of Morphometrics. Annual Review of Ecology and Systematics, 13, 451-470.

"Bookstein, F. (1990). Introduction and overview: geometry and biology. En J. Rohlf y F. Bookstein (Eds.), Proceedings of the Michigan morphometrics workshop (pp. 61-74). Ann Arbor: The University of Michigan Museum of Zoology.

»Bookstein, F. (1991). Morphometric tools for landmark data. Geometry and biology. Nueva York: Cambridge University Press.

》Bookstein, F. (1996-1997). Landmarks methods for form without landmarks: Morphometrics of group differences in outline shape. Medical Image analysis, 1(3), 225243.

» Buchanan, B. y Collard, M. (2010). A geometric morphometric-based assessment of blade shape differences among Paleoindian projectile point types from western North America. Journal of Archaeological Science, 37, 350-359.

" Buchanan, B., Collard, M., Hamilton, M. y O’Brien, M. (2011). Points and prey: a quantitative test of the hypothesis that prey sizes influences early Paleoindian projectile point form. Journal of Archaeological Science, 38, 852-864. DOI 10.1016/j.jas.2010.11.007

" Buchanan, B., Eren, M., Boulanger, M. y O’Brien, M. (2015). Size, Shape, Scars, and Spatial Patterning: A Quantitative Assessment of Late Pleistocene (Clovis) Point Resharpening. Journal of Archaeological Science: Reports, 3, 11-21.

》Cardillo, M. (2009). Temporal trends in the morphometric variation of the lithic projectile points during the middle Holocene of southern Andes (Puna region)-A coevolutionary approach. En H. J. Muscio y G. López (Eds.), Theoretical and methodological issues in Evolutionary Archaeology (pp. 13-20). Oxford: Archaeopress.

"Cardillo, M. (2010). Some applications of Geometric Morphometrics to Archaeology. En A. Elewa (Ed.), Morphometrics for nonmorphometricians (pp. 325-355). Verlag-BerlinHeidelberg: Springer. DOI 10.1007/978-3-540-95853-6

" Cardillo, M., Borrazzo, K. y Charlin, J. (2016). Environment, Space, and Morphological Variation of Projectile Points in Patagonia (Southern South America). Quaternary International, 422, 44-56. http://doi.org/10.1016/j.quaint.2015.11.134

" Cardillo, M. y Charlin, J. (2016). Morphological Diversification of Stemmed Projectile Points of Patagonia (Southernmost South America). Assessing Spatial Patterns by Means of Phylogenies and Comparative Methods. En R. lovita y K. Sano (Eds.), Multidisciplinary Approaches to the Study of Stone Age Weaponry (pp. 261-272). Dordrecht: Springer. DOI 10.1007/978-94-017-7602-8_18.

"Carpiceci, A. C. (2009). Arte e historia: Egipto 500 años de civilización. Florencia: Casa Editrice Bonechi. 
"Castiñeira, C., Cardillo, M., Charlin, J. y Baeza, J. (2011). Análisis de morfometría geométrica en puntas Cola de Pescado del Uruguay. Latin American Antiquity, 22(3), 335358.

" Castiñeira, C., Charlin, J., Cardillo, M. y Baeza, J. (2012). Exploring morphometric variations in Fishtail projectile points from Uruguay, Pampa and Patagonia. En L. Miotti, M. Salemme, N. Flegenheimer y T. Goebel (Eds.), Southbound: Late Pleistocene Peopling of Latin America (pp. 57-62). Texas: Center for the Study of the First Americans.

"Charlin, J. y Cardillo, M. (2018). Reduction constraints and shape convergence along tool ontogenetic trajectories. En M. O'Brien, B. Buchanan y M. Eren (Eds.), Convergent Evolution in Stone-Tool Technology (pp. 109-129). Cambridge: Vienna Series in Theoretical Biology. MIT Press.

»Charlin, J., Cardillo, M. y Borrazzo, K. (2014). Spatial Patterns in Late Holocene Lithic Projectile Point Technology of Tierra del Fuego (Southern South America): Assessing Size and Shape Changes. World Archaeology, 46(1), 78-100. http://dx.doi.or g/10.1080/00438243.2014.890914

"Charlin, J.y González-José, R. (2012). Size and shape variation in Late Holocene projectile points of southern Patagonia. A geometric morphometric study. American Antiquity, 77(2), 221-242.

»Charlin, J.y González-José, R. (2018). Testing an ethnographic analogy through geometric morphometrics: a comparison between ethnographic arrows and archaeological projectile points from Fuego-Patagonia. Journal of Anthropological Archaeology, 51, 159172.

» Charlin, J. y Hernández Llosas, M. I. (2016). Morfometría geométrica y representaciones rupestres: explorando las aplicaciones de los métodos basados en landmarks. Arqueología, 22(1), 103-125.

"Charlin, J. y Manzi, L. (2017). Estudio de las representaciones de Neferhotep en las paredes norte y sur de la TT49 a través de la morfometría geométrica. En A. Brancaglion Jr. y G. Chapot (Eds.), Estudos de Egiptologia IV (pp. 215-229). Río de Janeiro: Laboratório de Egiptologia do Museu Nacional.

»Charlin, J. y Manzi, L. (2018). Una aplicación de la morfometría geométrica al estudio de las variaciones morfológicas de los retratos de la tumba de Neferhotep -TT49-, Dinastía XVIII, Reino Nuevo, Egipto. Sociedades Precapitalistas, 8(1), e029. https://doi. org/10.24215/22505121e029

" Choisy, A. (2007). El arte de construir en Egipto. Madrid: Editorial Instituto Juan de Herrera.

»Cobden, R., Clarkson, C., Proce, G., Davis, B., Geneste, J. M., Delannoy, J. J., Barker, B., Lamb, L. y Gunn, R. (2017). The identification of extinct megafauna in rock art using geometric morphometrics: A Genyornis newtoni painting in Arnhem Land, northern Australia? Journal of Archaeological Science, 87, 95-107. https://doi.org/10.1016/j. jas.2017.09.013

» Davies, N. G. (1933). The Tomb of Neferhotep at Thebes. Nueva York: Metropolitan Museum of Art of New York.

" Fantechi, S. (2017). Arquitectura funeraria y repertorio iconográfico en la transición de la dinastía XVIII a la XIX. Sociedades Precapitalistas, 7(1), eo22. https://doi. org/10.24215/22505121eo22

»Fantechi, S. y Zingarelli, A. (2003). El bouquet de Amón y su simbología en TT49. Trabajo presentado en IX Jornadas Interescuelas/Departamentos de Historia. Córdoba, Argentina. 
»Fantechi, S. y Zingarelli, A. (2006). Muerte y renovación en el antiguo Egipto: las ofrendas florales. En Facultad de Filosofía y Letras (Ed.), Cuadernos de Ética, Estética y Religión II: El hombre ante la muerte (pp. 203-212). Tucumán: Universidad Nacional de Tucumán.

»Fantechi, S. y Zingarelli, A. (2009). El banquete funerario en las tumbas privadas tebanas de la dinastía XVIII. En C. Ames y M. Sagristani (Comps.), Actas de las Segundas Jornadas Nacionales de Historia Antigua / Primeras Jornadas Internacionales de Historia Antigua (pp. 111-118). Córdoba: Universidad Nacional de Córdoba.

» Faulkner, R. (1976). A Concise Dictionary of Middle Egyptian. Oxford: Griffith Institute.

» Gunz, P. y Mitteroecker, P. (2013). Semilandmarks: a method for quantifying curves and surfaces. Hystrix, the Italian Journal of Mammalogy, 24(1), 103-106.

» Hartwig, M. K. (2003). Style and Visual Rhetoric in Theban Tomb Painting. En Z. Hawas y L. Pinch Brock (Eds.), Egyptology at the Dawn of the Twenty-first Century. Proceeding of the Eight International Congress of Egyptologists, Cairo, 2000 (Volumen 2, pp. 298-307). CairoNueva York: The American University in Cairo Press.

» Hermoso Cuesta, M. (2008). Breve historia del arte egipcio. Barcelona: Montesinos.

»Katary, S. (2009). Distinguishing subclasses in New Kingdom society on evidence of the Wilbour Papyrus. En J. C. Moreno García (Ed.), Élites et pouvoir en Égypte ancienne (pp. 263-319). Cahiers de recherches de l'Institut de Papyrologie et Égyptologie de Lille 28.

»Kemp, B. (1977). The city of el-Amarna as a source for the study of urban society in ancient Egypt. World Archaeology, 9(2), 123-139. https://doi.org/10.1080/o0438243.1977.9979691

» Laboury, D. (2009). Royal portrait and ideology. Evolution and signification of the statuary of Thutmosis III. En E. Cline y D. O'Connor (Eds.), Thutmosis III. A New Biography (pp. 260-291). Ann Harbor: The University of Michigan Press.

» Laboury, D. (2010). Portrait versus Ideal Image. En W. Wendrich, J. Dieleman, E. Frood y J. Baines (Ed.), UCLA Encyclopedia of Egyptology (pp. 1-18). Los Angeles: Universidad de California. http://digital2.library.ucla.edu/viewltem.do?ark=21198/zzoo25jjvo.

»Lawing, A. y Polly, P. (2009). Geometric morphometrics: Recent applications to the study of evolution and development. Journal of Zoology, 28o, 1-7.

» Legendre, P. y Legendre, L. (1998). Numerical Ecology. 2da Edición. Elsevier.

»Lemos, R. (2017). Material culture and social interactions in New Kingdom non-elite cemeteries. En J. Chyla, J. Dębowska-Ludwin, K. Rosin'ska-Balik y C. Walsh (Eds), Current Research in Egyptology 2016: Proceedings of the Seventeenth Annual Symposium (pp. 121135). Oxford: Oxbow Books.

» Manniche, L. (1989 [1987]). The Tombs of the Nobles at Luxor. Cairo: The American University in Cairo Press.

» Manzi, L. (2016). La especificidad del retrato: un estudio comparativo. En A. Brancaglion y C. Alfieri Gama-Rolland (Eds.), Semna-Estudos de Egiptologia III (pp. 145-165). Río de Janeiro: Laboratório de Egiptologia do Museu Nacional.

» Marcus, L., Bello, E. y Garcia-Valdecasas, A. (Eds.) (1993). Contributions to Morphometrics. Madrid: Museo Nacional de Ciencias Naturales, Consejo Superior de Investigaciones Científicas.

» Mitteroecker, P. y Gunz, P. (2009). Advances in Geometric Morphometrics. Evolutionary Biology, 36, 235-247.

» Morales, J., Soto, M., Lorenzo C., y Vergès, J. (2015). The Evolution and Stability of Stone Tools: The effects of Different Mobility Scenarios in Tool Reduction and Shape Features. Journal of Archaeological Science: Reports, 3, 295-305. 
» Okumura, M. y Araujo, A. (2014). Long-Term Cultural Stability in Hunter-Gatherers: a Case Study Using Traditional and Geometric Morphometric Analysis of Lithic Stemmed Bifacial Points From Southern Brazil. Journal of Archaeological Science, 45, 59-71.

»Okumura, M. y Araujo, A. (2019). Archaeology, biology, and borrowing: A critical examination of Geometric Morphometrics in Archaeology. Journal of Archaeological Science, 101, 149-158.

»Parra Ortiz, J. M. (2011). El reino antiguo. En J. M. Parra Ortiz (Ed.), El Egipto Antiguo. Sociedad, Economía, Política (pp. 125-180). Madrid: Marcial Pons, Ediciones de Historia.

"Pérez, S. (2003). Morfometría de poblaciones humanas prehistóricas: Una comparación de técnicas Tradicionales y Geométricas. Intersecciones en Antropología, 4, 121-129.

"Pereyra, M. V. (2011a). El libro para salir al día. Buenos Aires: Dunken

»Pereyra, M. V. (2011b). El gran templo de Amón en la tumba de Neferhotep TT49. Revista del Instituto de Historia Antigua Oriental, 17, 17-26.

»Pereyra, M. V. (2012a) Espacio y tiempo ritual en la antigua Tebas. Consideraciones en torno a su representación (parte I). Mundo Antigo, 1(1), 68-87.

»Pereyra, M. V. (2012b). El palacio real en el umbral del Más Allá. Novos Trabalhos de Egiptologia lbérica, 1, 871-883.

"Pereyra, M. V. (2017). La tumba como "retrato", entre la construcción social y la individual. En B. Neméth (Ed.), Now Behold My Spacious Kingdom. Studies Presented to Zoltán Imre Fábián On the Occasion of His 63rd Birthday (pp. 82-95). Budapest: L'Harmattan.

"Pereyra, M. V., Alzogaray, N., Zingarelli, A., Fantechi, S., Vera, S., Verbeek, Ch., Brikmann, S. y Graue, B. (2006). Imágenes a preservar en la Tumba de Neferhotep (TT49). Tucumán: Universidad Nacional de Tucumán.

»Pereyra, M. V., Fantechi, S. y Zingarelli, A (2007). Figuration of an Egyptian Theban Tomb belonging to Ay's Reign: TT49. En J. C. Goyon y Ch. Cardin (Eds.), Proceedings of the Ninth International Congress of Egyptologists, Grenoble, 6-12 September 2004 (Volumen 2, pp. 1485-1494). Leuven-Paris-Dudley: Peeters.

"Pereyra, M. V., Manzi, L. y Charlin, J. (2016). Hetep di nesu y su lógica en el Egipto faraónico: la necrópolis tebana ca. 1550-1200 a.C. Trabajo presentado en XIX Congreso Nacional de Arqueología Argentina, San Miguel de Tucumán, Argentina.

»Pereyra, M. V. y Zingarelli, A. (2004) La celebración y el llanto en el pasaje al Más Allá. En: Publicación de las VII Jornadas Nacionales de Historia de las Mujeres y II Congreso Iberoamericano de Estudios de Género, Comisión de la Mujer, GESNOA, Universidad Nacional de Salta (CD ROM). Salta, Argentina.

" Rohlf, J. (1999). Shape statistics: procrustes superimpositions and tangent spaces. Journal of Classification, 16, 197-223.

"Rohlf, J. y Bookstein, F. (Eds.) (1990). Proceedings of the Michigan morphometrics workshop. Special Publication № 2. Ann Arbor: The University of Michigan Museum of zoology.

» Sapirstein, P. (2013). Painters, potters, and the scale of attic vase-painting industry. American Journal of Archaeology, 117(4), 493-510.

"Shott, M. y Trail, B. (2010). Exploring new approaches to lithic analysis: laser scanning and geometric morphometrics. Lithic Technology, 35(2), 195-220.

"Shott, M. y Trail, B. (2012). New Developments in Lithic Analysis Laser Scanning and Digital Modeling. The SAA Archaeological Record, 12-38.

"Slice, D. (Ed.) (2005). Modern morphometrics in Physical Anthropology. Chicago: Universidad de Chicago. 
» Slice, D. (2007). Geometric morphometrics. Annual Review of Anthropology, 36, 261-281. DOI: 10.1146/annurev.antrhro.34.081804.120613.

» Slice, D., Bookstein, F., Marcus, L. y Rolhf, J. (2009). A Glossary for Geometric Morphometrics. http://life.bio.sunysb.edu/morph/glossary/gloss1.html, http://life.bio. sunysb.edu/morph/glossary/gloss2.html (Acceso: 14 de julio de 2019).

»Spence, K. E. (2010). Settlement Structure and Social Interaction at El-Amarna. En M. Bietak, E. Czerny y I. Forstner-Müller (Eds.), Cities and Urbanism in Egypt (pp. 289-298). Vienna: Österreichische Akademie der Wissenschaften.

»Staring, N. (2008). Iconographic programme and tomb architecture: a focus on desertrelated themes. En V. Gashe y J. Finch (Eds.), Current Research in Egyptology. Proceedings of the Nineth Annual Symposium University of Manchester. Bolton: Rutherford.

» Thulman, D. (2012). Discriminating Paleoindian point types from Florida using landmark geometric morphometrics. Journal of Archaeological Science, 39, 1599-1607.

»Viscosi, V.yCardini, A. (2011). Leafmorphology, taxonomyand geometric morphometrics: A simplified protocol for beginners. PLOS ONE, 6(10), e25630. doi:10.1371/journal. pone.0025630

»Webstern, M. y Sheets, H. (2010). A practical introduction to landmark-based geometric morphometrics. En J. Alroy y G. Hunt (Eds.), Quantitative Methods in Paleobiology (pp. 163-188). Cambridge: The Paleontological Society Papers, Volumen 16.

»Yomaha, S. (2009). La tumba como espacio ritual. XII Jornadas Interescuelas/ Departamentos de Historia. Departamento de Historia, Facultad de Humanidades y Centro Regional Universitario Bariloche. San Carlos de Bariloche: Universidad Nacional del Comahue.

» Zelditch, M., Swiderski, D., Sheets, H. y David, H. (2014). A Practical Companion for Geometric Morphometrics for Biologists: Running analyses infreely-available software. http://booksite.elsevier.com/9780123869036/content/Workbook.pdf.

» Zelditch, M., Swiderski, D., Sheets H. y Fink, W. (2004). Geometric Morphometrics for Biologists: A primer. Londres: Elsevier Academic Press.

»Zingarelli, A. (2005). Some considerations about the water offered (poured) by the tree goddess at TT49. En A. Amenta, M. M. Luiselli y M. N. Sordi. (Eds.), L'acqua nell'antico Egitto: vita, rigenerazione, incantesimo, medicamento: First International Conference or Young Egyptologists (pp. 381-388). Roma: L’Erma di Bretschneider.

»Zingarelli, A. y Fantechi, S. (2014). Iconografía e iconología de la unión familiar y social en el Más Allá (Tebas-Reino Nuevo). En C. Onaha y L. Rodríguez de la Vega (Eds.), Colección -ALADAA. (pp. 147-157). La Plata: Asociación Latinoamericana de Estudios de Asia y África. 